\title{
Correction to: Biomarkers associating endothelial dysregulation in pediatric-onset systemic lupus erythematous
}

Wan-Fang Lee ${ }^{1}$, Chao-Yi Wu ${ }^{1,2}$, Huang-Yu Yang ${ }^{2,3}$, Wen-I Lee ${ }^{1}$, Li-Chen Chen ${ }^{1}$, Liang-Shiou Ou ${ }^{1}$ and Jing-Long Huang ${ }^{1,2^{*}}$

\section{Correction to: Pediatr Rheumatol Online J (2019) 17:69} https://doi.org/10.1186/s12969-019-0369-7

Following publication of the original article [1], we have been notified that the colour representation of the graph is not correct in Figure 6 legend.

The legend of Figure 6 should state the below:

Fig. 6 Predictive Value of Biomarkers in Renal involvement Compared to Anti-dsDNA. The ROC curve of these markers compared to Anti-dsDNA in renal involvement in pSLE patients. Red: Thrombomodulin. Light Blue: Anti-dsDNA

\footnotetext{
Author details

${ }^{1}$ Division of Allergy, Asthma, and Rheumatology, Department of Pediatrics, Chang Gung Memorial Hospital Linko branch, Taoyuan, Taiwan. ${ }^{2}$ Chang Gung University, College of Medicine, Taoyuan, Taiwan. ${ }^{3}$ Department of Nephrology, Chang Gung Memorial Hospital Linko branch, Taoyuan, Taiwan.
}

Published online: 18 February 2020

\section{Reference}

1. Lee WF, et al. Biomarkers associating endothelial Dysregulation in pediatriconset systemic lupus erythematous. Pediatr Rheumatol Online J. 2019;17:69. https://doi.org/10.1186/s12969-019-0369-7.

*Correspondence: long@adm.cgmh.org.tw

${ }^{1}$ Division of Allergy, Asthma, and Rheumatology, Department of Pediatrics,

Chang Gung Memorial Hospital Linko branch, Taoyuan, Taiwan

${ }^{2}$ Chang Gung University, College of Medicine, Taoyuan, Taiwan

Full list of author information is available at the end of the article

(c) The Author(s). 2020 Open Access This article is distributed under the terms of the Creative Commons Attribution 4.0 International License (http://creativecommons.org/licenses/by/4.0/), which permits unrestricted use, distribution, and reproduction in any medium, provided you give appropriate credit to the original author(s) and the source, provide a link to the Creative Commons license, and indicate if changes were made. The Creative Commons Public Domain Dedication waiver (http://creativecommons.org/publicdomain/zero/1.0/) applies to the data made available in this article, unless otherwise stated. 